\title{
Adsorption of Polystyrene onto Silica at the Theta Temperature
}

\author{
Masami Kawaguchi, Kazuhisa Hayakawa, and Akira TaKahashi \\ The Chemistry Department of Industry and Resources, Faculty of Engineering, \\ Mie University, Tsu, Mie 514, Japan.
}

(Received December 7, 1979)

\begin{abstract}
Adsorption of polystyrene having a molecular weight ranging from 600 to 2,000,000 onto Aerosil 130 from cyclohexane solutions at the theta temperature was studied by UV and IR spectroscopy. Adsorbance, $(A)$ was determined using UV spectroscopy while both the fraction of the surface sites occupied, $(\Sigma)$ and the fraction of the segments adsorbed per polymer chain, $(p)$ were measured by IR spectroscopy. Adsorbance increases with increasing molecular weight up to $M_{w}=50 \times 10^{4}$ and then levels off. The value of $\Sigma$ increases while the $p$ value decreases with increasing molecular weight. The close correlation between the adsorbed amount $\Gamma$ per site determined UV spectroscopy and the ratio $\Sigma / p$ measured by IR spectroscopy can be observed except for cases involving high molecular weight samples. The data were compared with the theories of Scheutjens and Fleer and Silberberg based on a lattice model. There was excellent agreement between the data and these theories.

KEY WORDS Adsorption / Polystyrene / Silica / UV Spectroscopy / IR

Spectroscopy / Adsorbance / Surface Sites / Adsorbed Segments /
\end{abstract}

The major confronted central problems in the study of polymer adsorption onto solid-surface are clarification of the conformation of the adsorbed polymer chains and the polymer-solvent, polymerpolymer, and polymer-substrate interactions as well. In these problems, not only the extension of the adsorbed polymer layer, $t$ but also the adsorbance, $A$ $\left(\mathrm{gcm}^{-2}\right)$ are crucially important. Besides the adsorbance, the fraction of the sites occupied on the solid surface, $\Sigma$ and the fraction of the segments adsorbed per polymer chain, $p$ are also necessary for elucidating the conformation of the adsorbed polymer chain. Theories developed since 1968 are detailed in their prediction of the molecular weight dependence of the adsorbance, $A$, the fraction of the sites occupied, $\Sigma$, and the fraction of the segments adsorbed, $p .^{1-9}$ No unique experimental method indicating the extension of adsorbed polymer layer, $t$, the adsorbance, $A$, the fraction of the sites occupied, $\Sigma$, and the fraction of the segments adsorbed, $p$ simultaneously is available at the present stage. Ellipsometry ${ }^{10-14}$ provides the information of the adsorbance, $A$ and the extension of adsorbed polymer layer, $t$ but not the fraction of the sites occupied, $\Sigma$ and the fraction of the segments adsorbed, $p$. On the other hand, spectroscopy ${ }^{15-17}$ can offer the adsorbance, $A$, the fraction of the sites occupied, $\Sigma$ is unity and claimed that their data are in quantitative agreement with the theory of Silberberg ${ }^{1}$ which is a loop-train model, and spectroscopy except for the problem of the extension of the adsorbed polymer.

Recently, Linden and Leemput ${ }^{17}$ carried out the adsorption of polystyrene onto Aerosil silica particles from cyclohexane at $35^{\circ} \mathrm{C}$, using IR spectroscopy. They found that the fraction of sites occupied, $\Sigma$ is unity and claimed that their data is in quantitative agreement with the theory of Siliberberg ${ }^{1}$ which is a loop-train model, and neglected the contribution from the tails. We have carried out an adsorption study on the same system and determined the adsorbance $A$, the fraction of the sites occupied, $\Sigma$, and the fraction of the segments adsorbed, $p$ by using UV and IR spectroscopy; however our data indicating the fraction of the sites occupied, $\Sigma$ is at variance with their findings. Moreover, very recently, Scheutjens and Fleer ${ }^{9}$ developed a theory based on a loop-train-tail model. In this paper, we intend to compare our data with the adsorption theories of Silberberg as well as that of 
Scheutjens and Fleer.

\section{EXPERIMENTAL}

\section{Materials}

The linear polystyrene samples, having a narrow molecular weight distribution, were supplied by ArRo Laboratories Inc. (A Series) and Pressure Chemical Co. (P Series). The characteristics of the eight polystyrene samples are given in Table I. Firstgrade cyclohexane was distilled, passed through a silica gel column to remove aromatic impurities, refluxed over sodium metal and then distilled fractionally just before use. All polystyrene cyclohexane solutions were filtered through a millipore HA filter prior to measurements.

Table I. Polymer characterization data

\begin{tabular}{crc}
\hline Samples $^{\mathrm{a}}$ & $M_{w} \times 10^{-3}$ & $M_{w} / M_{n}$ \\
\hline A-1 & 0.6 & 1.07 \\
A-2 & 4.0 & 1.06 \\
A-3 & 9.0 & 1.05 \\
P-1 & 37.0 & 1.06 \\
P-2 & 110.0 & 1.06 \\
P-3 & 498.0 & 1.07 \\
P-4 & 670.0 & 1.06 \\
P-5 & 2000.0 & 1.06 \\
\hline
\end{tabular}

a The A series were supplied by ArRo Laboratories Inc. and the $\mathrm{P}$ series were supplied by Pressure Chemical Co.

The nonporous silica Aerosil 130 supplied by Degussa A. G. was used as the adsorbent. According to the manufacturer, the particle diameter was $16 \mathrm{~nm}$ with a surface area of $141 \mathrm{~m}^{2} \mathrm{~g}^{-1}$. This material was heated at $250^{\circ} \mathrm{C}$ for $18 \mathrm{~h}$ under vacuum to ensure an average-surface-site density close to three $\mathrm{OH}$ groups per $100 \AA^{2} .^{17}$

\section{Measurement Techniques}

Adsorbance $(A)$. Adsorbance, $A\left(\mathrm{gcm}^{-2}\right)$ per unit area was determined indirectly from the concentration change in the supernatant solution. Samples of silica suspensions containing polymer were removed from a glass vessel following one day of equilibrium at $35^{\circ} \mathrm{C}$. The samples were centrifuged for 5 minutes at $5,000 \mathrm{~g}$ to settle the solid completely. In a UV spectroscopy (Union Giken System 77-1) provided with a glass single cell of matched $10 \mathrm{~mm}$ path lengths, thermostatted in a water bath at $35^{\circ} \mathrm{C}$, the intensities of the supernatant solutions were measured at $\lambda=263 \mathrm{~nm}$. The relevent concentrations were determined from a calibration curve.

Fraction of the Surface Sites Occupied $(\Sigma)$. On centrifuging the equilibrium adsorption mixtures, the silica particles were settled as a stable gel. A portion of the gel (usually about $0.025 \mathrm{~g}$ ) was then weighted onto the polished face of a $13 \mathrm{~mm}$ diameter of IR $\mathrm{KBr}$ cell. A part of supernatant solution was put into an identical reference cell. Infrared measurements were performed on a Nihon Bunko Model IR-G spectrometer equipped with two path length cells at $35^{\circ} \mathrm{C}$.

The differential infrared spectra obtained between a cell containing only the supernatant polymer solution and a cell containing the silica gel on which polymer was adsorbed are shown in Figures 1a, 1b, and $1 \mathrm{c}$ for samples of A-1, A-2, and P-3. A band $3680 \mathrm{~cm}^{-1}$ is assigned to the isolated silanols, while a band located $3588 \mathrm{~cm}^{-1}$ is assigned to the attachment of the phenyl groups of polystyrene chains on the isolated silanols. Thus, the number of free sites $\left(S_{3680}\right)$ and that of the occupied sites by phenyl groups $\left(S_{3588}\right)$ can be known using respective extinction coefficients; the extinction coefficient at

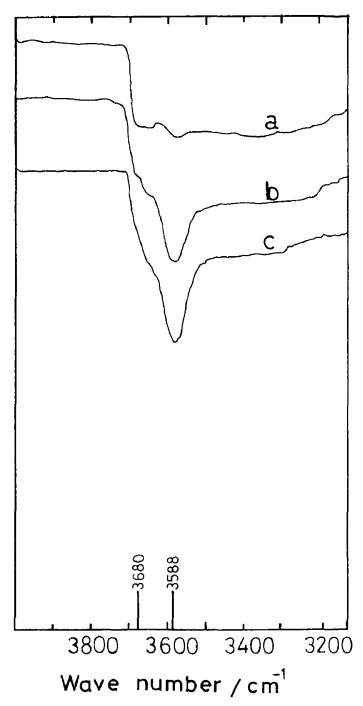

Figure 1. Infrared spectra of Aerosil 130 in cyclohexane, in the presence of polystyrene samples of various molecular weights: (a) A-1; (b) A-2; (c) P-3. 


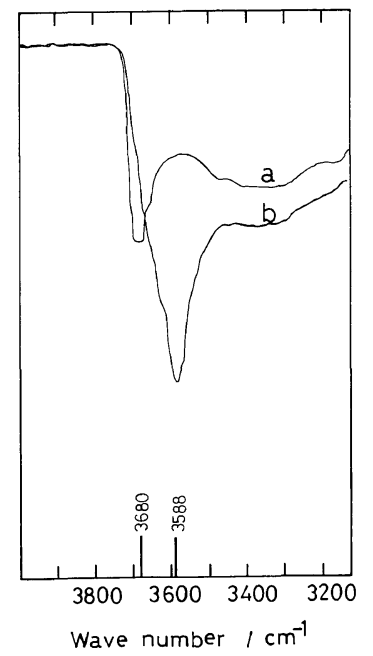

Figure 2. Infrared spectra taken on a silica gel immersed in (a) cyclohexane and (b) ethyl benzene.

$3680 \mathrm{~cm}^{-1}$ on silica gel immersed in cyclohexane was determined to be $55.61 \mathrm{~mol}^{-1} \mathrm{~cm}^{-1}$ (Figure 2a). At $3588 \mathrm{~cm}^{-1}$ the extinction coefficient for silica gel in ethyl benzene was obtained at $115.11 \mathrm{~mol}^{-1} \mathrm{~cm}^{-1}$ (Figure 2b). The fraction of the surface sites occupied, $\Sigma$ can be calculated from the following equation, using the value of $S_{3680}$ and $S_{3588}$,

$$
\Sigma=S_{3588} /\left(S_{3588}+S_{3680}\right)
$$

Fraction of the segments adsorbed ( $p)$. The fraction of the segments adsorbed, $p$ can be calculated from the following equation derived by Fontana and Thomas. ${ }^{15}$

$$
p=\frac{\text { (wt. of attached polymer segments } \mathrm{cm}^{-2} \text { ) }}{\text { (wt. of total adsorbed polymer } \mathrm{cm}^{-2} \text { ) }}
$$

The numerator in eq 2 is obtained from the product of the number of the sites occupied by phenyl groups and the molecular weight of a styrene monomer unit $M_{\mathrm{u}}$, whereas the denominator in eq 2 corresponds to the adsorbance, $A$.

\section{RESULTS}

\section{Adsorbance}

Adsorption isotherms are shown in Figure 3. The adsorption isotherms display the typical shape with a limiting plateau starting at a low bulk equilibrium

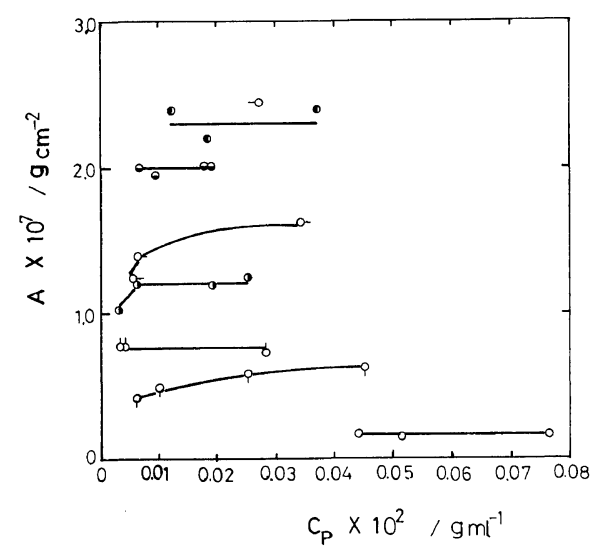

Figure 3. Adsorption isotherms of polystyrene: $\bigcirc, A-$ $1 ; \bigcirc, \mathrm{A}-2 ; \mathrm{O}, \mathrm{A}-3 ; \boldsymbol{O}, \mathrm{P}-1 ; \bigcirc-, \mathrm{P}-2 ; \bigcirc, \mathrm{P}-3 ;-\mathrm{O}, \mathrm{P}-4 ; \mathbf{O}$, P-5.

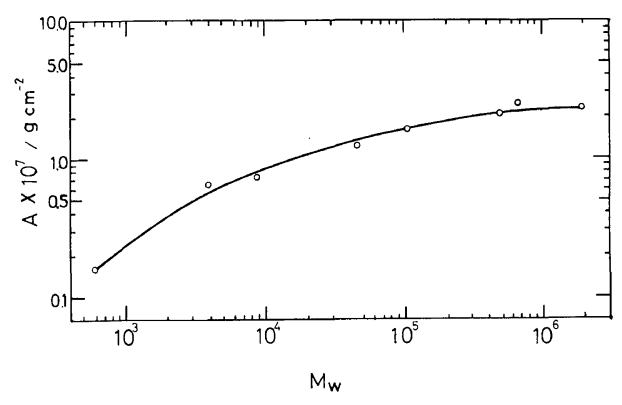

Figure 4. Logarithmic plot of the adsorbance at the highest bulk equilibrium concentration $v s$. the molecular weight.

concentration below $0.0001 \mathrm{~g} \mathrm{ml}^{-1}$ except for a sample of A-1. The adsorbance data and the bulk equilibrium concentration are summarized in Table II. In Figure 4, for respective polymer samples, the adsorbance obtained at the highest bulk equilibrium concentration is plotted against the molecular weight. At first, the adsorbance increases gradually with the molecular weight up to $M_{w}=49.8 \times 10^{4}$ and then levels off.

\section{Fraction of Surface Sites Occupied}

The fraction of the surface sites occupied, $\Sigma$, calculated from eq 1, is listed in Table II. The value of $\Sigma$ obtained at the highest bulk equilibrium concentration is plotted against the molecular weight 
M. Kawaguchi, K. Hayakawa, and A. Takahashi

Table II. Adsorption data of polystyrenes onto Aerosil 130

\begin{tabular}{|c|c|c|c|c|c|c|}
\hline \multirow[t]{2}{*}{$\begin{array}{l}\text { Polymer } \\
\text { samples }\end{array}$} & \multirow{2}{*}{$\begin{array}{c}\text { Bulk } \\
\text { equilibrium } \\
\text { concentration, } \\
C_{\mathrm{p}} \times 10^{2}\end{array}$} & \multirow{2}{*}{$\begin{array}{l}\text { Adsorbance, } \\
\qquad A \times 10^{7} \\
\mathrm{gcm}^{-2}\end{array}$} & \multirow{2}{*}{$\begin{array}{c}\text { Fraction of } \\
\text { the segments } \\
\text { adsorbed, } \\
p\end{array}$} & \multirow{2}{*}{$\begin{array}{c}\text { Fraction of } \\
\text { the sites } \\
\text { occupied, } \\
\Sigma\end{array}$} & \multicolumn{2}{|c|}{ Adsorbed amount } \\
\hline & & & & & $\Gamma$ & $\Sigma / p$ \\
\hline A-1 & $\begin{array}{l}0.044 \\
0.051 \\
0.076\end{array}$ & $\begin{array}{l}0.17 \\
0.15 \\
0.16\end{array}$ & $\begin{array}{l}1.00 \pm 0.10 \\
0.98 \pm 0.10 \\
0.99 \pm 0.10\end{array}$ & $\begin{array}{l}0.36 \pm 0.04 \\
0.30 \pm 0.03 \\
0.32 \pm 0.03\end{array}$ & $\begin{array}{l}0.33 \\
0.29 \\
0.31\end{array}$ & $\begin{array}{l}0.36 \pm 0.04 \\
0.31 \pm 0.03 \\
0.32 \pm 0.03\end{array}$ \\
\hline A-2 & $\begin{array}{l}0.0059 \\
0.0098 \\
0.025 \\
0.040\end{array}$ & $\begin{array}{l}0.43 \\
0.50 \\
0.59 \\
0.63\end{array}$ & $\begin{array}{l}0.70 \pm 0.07 \\
0.93 \pm 0.09 \\
0.59 \pm 0.06 \\
0.56 \pm 0.06\end{array}$ & $\begin{array}{l}0.53 \pm 0.05 \\
0.56 \pm 0.06 \\
0.51 \pm 0.05 \\
0.45 \pm 0.05\end{array}$ & $\begin{array}{l}0.83 \\
0.97 \\
1.14 \\
1.22\end{array}$ & $\begin{array}{l}0.76 \pm 0.08 \\
0.60 \pm 0.07 \\
0.86 \pm 0.09 \\
0.80 \pm 0.08\end{array}$ \\
\hline A-3 & $\begin{array}{l}0.0032 \\
0.0042 \\
0.028\end{array}$ & $\begin{array}{l}0.78 \\
0.79 \\
0.74\end{array}$ & $\begin{array}{l}0.41 \pm 0.04 \\
0.38 \pm 0.04 \\
0.47 \pm 0.05\end{array}$ & $\begin{array}{l}0.46 \pm 0.05 \\
0.43 \pm 0.04 \\
0.53 \pm 0.05\end{array}$ & $\begin{array}{l}1.51 \\
1.52 \\
1.43\end{array}$ & $\begin{array}{l}1.12 \pm 0.11 \\
1.13 \pm 0.11 \\
1.13 \pm 0.11\end{array}$ \\
\hline P-1 & $\begin{array}{l}0.0029 \\
0.0063 \\
0.019 \\
0.025\end{array}$ & $\begin{array}{l}1.03 \\
1.20 \\
1.20 \\
1.25\end{array}$ & $\begin{array}{l}0.33 \pm 0.03 \\
0.30 \pm 0.03 \\
0.29 \pm 0.03 \\
0.26 \pm 0.03\end{array}$ & $\begin{array}{l}0.79 \pm 0.08 \\
0.62 \pm 0.06 \\
0.68 \pm 0.07 \\
0.69 \pm 0.07\end{array}$ & $\begin{array}{l}1.99 \\
2.32 \\
2.32 \\
2.41\end{array}$ & $\begin{array}{l}2.39 \pm 0.24 \\
2.07 \pm 0.21 \\
2.34 \pm 0.23 \\
2.65 \pm 0.27\end{array}$ \\
\hline P-2 & $\begin{array}{l}0.0054 \\
0.0063 \\
0.034\end{array}$ & $\begin{array}{l}1.24 \\
1.40 \\
1.62\end{array}$ & $\begin{array}{l}0.21 \pm 0.02 \\
0.25 \pm 0.03 \\
0.21 \pm 0.02\end{array}$ & $\begin{array}{l}1.0 \pm 0.10 \\
1.0 \pm 0.10 \\
0.59 \pm 0.06\end{array}$ & $\begin{array}{l}2.39 \\
2.70 \\
3.13\end{array}$ & $\begin{array}{l}4.76 \pm 0.48 \\
4.00 \pm 0.40 \\
2.80 \pm 0.28\end{array}$ \\
\hline P-3 & $\begin{array}{l}0.0092 \\
0.0065 \\
0.018 \\
0.019\end{array}$ & $\begin{array}{l}1.95 \\
2.00 \\
2.20 \\
2.10\end{array}$ & $\begin{array}{l}0.21 \pm 0.02 \\
0.23 \pm 0.02 \\
0.14 \pm 0.01 \\
0.18 \pm 0.02\end{array}$ & $\begin{array}{l}1.0 \pm 0.10 \\
1.0 \pm 0.10 \\
0.73 \pm 0.07 \\
0.70 \pm 0.07\end{array}$ & $\begin{array}{l}3.76 \\
3.86 \\
4.24 \\
4.05\end{array}$ & $\begin{array}{l}4.76 \pm 0.48 \\
4.34 \pm 0.43 \\
5.21 \pm 0.52 \\
3.89 \pm 0.39\end{array}$ \\
\hline P-4 & 0.027 & 2.45 & $0.11 \pm 0.01$ & $0.88 \pm 0.09$ & 4.82 & $8.00 \pm 0.80$ \\
\hline P-5 & $\begin{array}{l}0.012 \\
0.018 \\
0.037\end{array}$ & $\begin{array}{l}2.40 \\
2.20 \\
2.40\end{array}$ & $\begin{array}{l}0.22 \pm 0.02 \\
0.13 \pm 0.01 \\
0.12 \pm 0.01\end{array}$ & $\begin{array}{l}1.0 \pm 0.10 \\
1.0 \pm 0.10 \\
1.0 \pm 0.10\end{array}$ & $\begin{array}{l}4.63 \\
4.23 \\
4.63\end{array}$ & $\begin{array}{l}4.55 \pm 0.46 \\
7.69 \pm 0.77 \\
8.33 \pm 0.83\end{array}$ \\
\hline
\end{tabular}

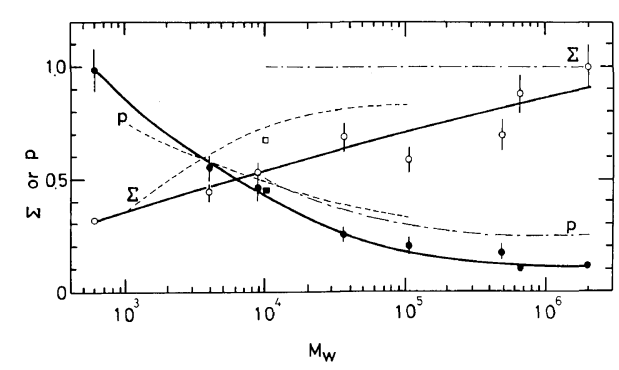

Figure 5. Semilogarithmic plots of the fraction of the surface sites occupied, $(\bigcirc, \Sigma)$ and the fraction of the segments adsorbed, $(\boldsymbol{O}, p)$ vs. the molecular weight. Dependences predicted at the theta temperature: (- - - ), from Scheutjens and Fleer's theory for the polymersubstrate interaction parameter $\chi_{\mathrm{s}}=1.3$ and the volume fraction of polymer, $\phi^{*}=10^{-3} ;\left(--\_--\right)$, from Silberberg's theory for the conformational parameter $\left(\gamma_{\mathrm{B}} \gamma_{\mathrm{s}}\right)=0.1, \chi_{\mathrm{s}}=\infty$, and $\phi^{*}=10^{-3} ;(\square, \Sigma ; \boldsymbol{\square}, p)$ from Silberberg's theory for $\left(\gamma_{\mathrm{B}} \gamma_{\mathrm{s}}\right)=0.1, \chi_{\mathrm{s}}=1.3$, and $\phi^{*}=10^{-3}$. in Figure 5 and increases with the molecular weight. The $\Sigma$ value usually decreases with increasing bulk equilibrium concentration as seen from Table II.

\section{Fraction of Segments Adsorbed}

The fraction of the segments adsorbed, $p$, calculated from eq 2, is listed in Table II and decreases with increasing bulk equilibrium concentration. The $p$ value at the highest bulk equilibrium concentration decreases with increasing molecular weight up to $M_{w}=11 \times 10^{4}$ and reaches a limiting value of $0.12-0.18$ as seen from Figure 5.

\section{DISCUSSION}

\section{Molecular Weight Dependence of the Adsorbed Amount}

Adsorbance, $A$, is commonly expressed in milligrams of polymer per weight (or better unit 
area) of the adsorbent. However, the adsorbed amount, $\Gamma$, corresponding to the total number of the segments adsorbed per site is actually the quantity of interest. Estimation of this quantity, $\Gamma$, is expected to clarify the conformation of the adsorbed polymer chain. The important quantity, $\Gamma$, is defined by,

$$
\Gamma=\left(A N_{\mathrm{A}}\right) /\left(n_{\mathrm{OH}} M_{\mathrm{u}}\right)
$$

where $A$ is the adsorbance $\left(\mathrm{g} \mathrm{cm}^{-2}\right), N_{\mathrm{A}}$ is Avogadro's number, $n_{\mathrm{OH}}$ is the number of silanol sites per unit area, equal to $3 \times 10^{14} \mathrm{OH}$ groups $\mathrm{cm}^{-2}$, and $M_{\mathrm{u}}$ is the molecular weight of a styrene monomer unit. The $\Gamma$ so defined is suitable for comparison with the theoretical $\Gamma$, based on a lattice model since it is regarded as the adsorbed amount per lattice site. In this respect, Aerosil Silica 130, used in this experiment, behaves as a suitable adsorbent since the number of site per unit area is known. $\Gamma$ is also difined as follows,

$$
\Gamma=\Sigma / p
$$

In Table II, $\Gamma$ obtained by eq 3 is compared with $\Sigma / p$ and the $\Sigma / p$ is plotted against $\Gamma$ in Figure 6. Except for the higher molecular weight fractions of P-4 and $P-5$, eq 4 is satisfied and indicates that the independent measurements of $\Gamma$ are in good agreement. The reason for the large deviations of points for P-4 and P-5 from the diagonal may be attributed to the large uncertainty in IR spectroscopy hampered by unstable gels obtained for P-4 and P-5 samples.

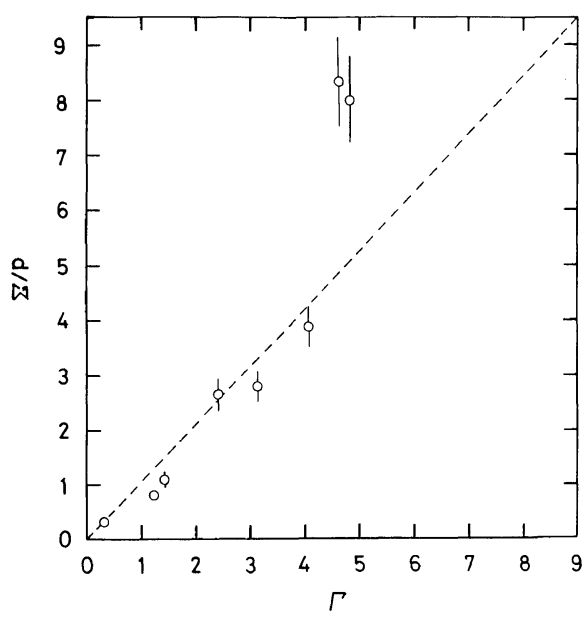

Figure 6. Plot of the ratio $\Sigma / p$ vs. $\Gamma$.
The experimental $\Sigma$ and $p$ values are compared with Scheutjens and Fleer as well as Silberberg's theories in Figure 5. As for the analysis of these theories, the published results of these authors have been used, although this procedure is far more exacting and difficult. In Scheutjens and Fleer's theory, the polymer-substrate interaction parameter, $\chi_{\mathrm{s}}=1.3$, the Flory-Huggins parameter, $\chi=0.5$ and the volume fraction of polymer, $\phi^{*}=10^{-3}$, which is close to the highest equilibrium concentration for respective samples. These values were selected for use in our experiments. Both the theoretical $\Sigma$ and $p$ are plotted in Figure 5 and fair agreement can be observed. In Silberberg's line, only at the molecular weight of $10^{4}$ comparison was made, assuming that the conformational parameter $\left(\gamma_{\mathrm{B}} \gamma_{\mathrm{s}}\right)=0.1, \quad \chi_{\mathrm{s}}=1.3, \quad \chi=0.5, \quad$ and $\phi^{*}=10^{-3}$. However, no appreciable difference between these theories has been observed. At the higher molecular weight region, $\chi_{\mathrm{s}}=\infty$ is assumed in Silberberg's theory, with the result that $\Sigma=1$, which is near the experimental point. As comparison shows, the decision for theory preference is difficult, since both theories can quantitatively explain the experimental $\Sigma$ and $p$. However, it appears that $\chi_{\mathrm{s}}=\infty$ is too extreme.

At the lowest molecular weight, the degree of polymerization is 6 and the adsorbed oligostyrene lies flat on the surface, since $p=1$.

The adsorbed amount, $\Gamma$, determined by eq 3 at the highest bulk equilibrium concentration, is plotted against the molecular weight in Figure 7. In Figure 7, comparison is also made with the theory of Scheutjens and Fleer on choosing $\chi_{\mathrm{s}}=1.3, \chi=0.5$, and $\phi^{*}=10^{-3}$. One must remember that the theory of Scheutjens and Fleer is valid only for polymerization lower than $10^{3}$, i.e., the region $M<10^{5}$. It was found that the $\Gamma$ calculated by Scheutjens and Fleer's theory is in excellent agreement with the observed $\Gamma$. For molecular weights above $10^{4}$, data was compared with that by Silberberg's theory. The adsorbed amount, $\Gamma$, calculated by Silberberg's theory for $\left(\gamma_{\mathrm{B}} \gamma_{\mathrm{s}}\right)=0.1$, $\chi_{\mathrm{s}}=\infty, \chi=0.5$, and $\phi^{*}=10^{-3}$, is also shown in Figure 7. In addition, only at a molecular weight of $10^{4}$, the value of $\Gamma$ calculated by the theory of Silberberg for $\left(\gamma_{\mathrm{B}} \gamma_{\mathrm{s}}\right)=0.1, \chi_{\mathrm{s}}=1.3, \chi=0.5$, and $\phi^{*}=10^{-3}$ is shown by a square in Figure 7. It is clear that the calculated $\Gamma$ from the theory of Silberberg is also nearly equal to the observed $\Gamma$. 


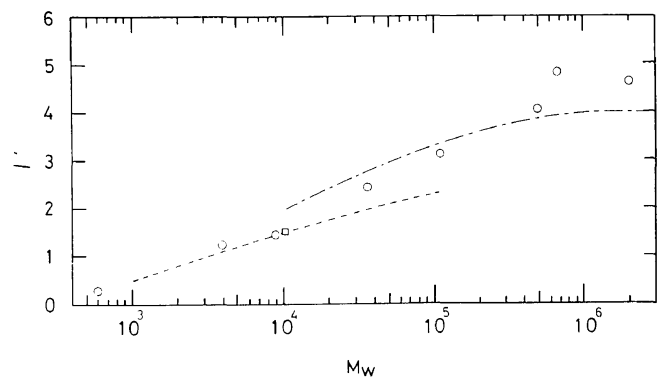

Figure 7. Semilogarithmic plot of the experimental adsorbed amount, $\Gamma$ vs. the molecular weight. Dependences predicted at the theta temperature: (-- $)$, from Scheutjens and Fleer's theory for $\chi_{\mathrm{s}}=1.3$ and $\phi^{*}=10^{-3} ;(-----)$, from Silberberg's theory for $\left(\gamma_{\mathrm{B}} \gamma_{\mathrm{s}}\right)=0.1, \chi_{\mathrm{s}}=\infty$, and $\phi^{*}=10^{-3} ;(\square)$, from Silberberg's theory for $\left(\gamma_{\mathrm{B}} \gamma_{\mathrm{s}}\right)=0.1, \chi_{\mathrm{s}}=1.3$, and $\phi^{*}=10^{-3}$.

In conclusion, both theories predict the experimental observation quantitatively. However, the conformation considered in both theories is different. In Silberberg's line, a loop-train conformation is assumed while Scheutjens and Fleer's theory is based on the loop-train-tail model. Thus, we can not conclude which conformation is predominant from the data of the adsorbance, fraction of the surface sites occupied, $\Sigma$, and fraction of the adsorbed segments, $p$ without relying upon the data concerning the extension of the adsorbed polymer.

Acknowledgement. This work was supported by a Grant-in-Aid for Scientific Research No. 247082 from the Ministry of Education, Science and Culture of Japan.

\section{REFERENCES}

1. A. Silberberg, J. Chem. Phys., 48, 2835 (1968).

2. K. Motomura and R. Matsuura, J. Chem. Phys., 50, 1281 (1969).

3. M. Lax, Macromolecules, 7, 660 (1974).

4. D. Chan, D. T. Mitchell, B. W. Ninham, and L. R. White, J. Chem. Soc. Faraday Trans. 2, 71 (1975).

5. R. J. Roe, J. Chem. Phys., 60, 4192 (1974).

6. R. J. Roe, J. Chem. Phys., 62, 490 (1975).

7. M. Lal and R. J. T. Stepto, J. Polym. Sci., Polym. Symp., No. 61, 401 (1977).

8. C. A. J. Hoeve, J. Polym. Sci., Polym. Symp., No. 61, 389 (1977).

9. J. M. H. M. Scheutjens, and G. J. Fleer, J. Phys. Chem., 83, 1619 (1979).

10. R. R. Stromberg, D. J. Tutas, and E. Passaglia, J. Phys. Chem., 69, 3955 (1965).

11. H. Gebhard and E. Killmann, Angew. Makromol. Chem., 53, 171 (1976).

12. A. Takahashi, M. Kawaguchi, and T. Kato, "Adhesion and Adsorption of Polymers," Part B, Polymer Science and Technology Series 12B, Plenum, New York, N.Y., 1980, p 729.

13. M. Kawaguchi and A. Takahashi, J. Polym. Sci., Polym. Phys. Ed., in press.

14. A. Takahashi, M. Kawaguchi, H. Hirota, and T. Kato, Macromolecules, in press.

15. B. J. Fontana and J. R. Thomas, J. Phys. Chem., 65, 480 (1961).

16. G. R. Joppien, Makromol. Chem., 175, 1931 (1974).

17. C. V. Linden and R. V. Leemput, J. Colloid Interface Sci., 67, 48 (1978). 